\title{
Bistatic Radar Coincidence Imaging Based on Multiple Measurement Vectors for Rotating Cone-Shaped Targets
}

\author{
Rui Li, ${ }^{1}$ Ying Luo $\mathbb{D}^{1,2,3}$ Qun Zhang, ${ }^{1,2,3}$ Yijun Chen, ${ }^{4}$ and Jia Liang ${ }^{1}$ \\ ${ }^{1}$ Institute of Information and Navigation, Air Force Engineering University, Xi'an 710077, China \\ ${ }^{2}$ Key Laboratory for Information Science of Electromagnetic Waves (Ministry of Education), Fudan University, \\ Shanghai 200433, China \\ ${ }^{3}$ Collaborative Innovation Center of Information Sensing and Understanding, Xi'an 710077, China \\ ${ }^{4}$ College of Information Engineering, Engineering University of PAP, Xi'an 710086, China \\ Correspondence should be addressed to Ying Luo; luoying2002521@163.com
}

Received 2 January 2020; Revised 3 July 2020; Accepted 6 July 2020; Published 13 August 2020

Academic Editor: Giovanni Diraco

Copyright (C) 2020 Rui Li et al. This is an open access article distributed under the Creative Commons Attribution License, which permits unrestricted use, distribution, and reproduction in any medium, provided the original work is properly cited.

\begin{abstract}
Bistatic radar imaging can overcome limitations of monostatic radar imaging and obtain abundant target feature information; thus, it is followed with interest. Different from bistatic inverse synthetic aperture radar (Bi-ISAR) imaging, bistatic radar coincidence imaging (Bi-RCI) provides a new tack on the bistatic radar imaging technique. In this paper, a Bi-RCI based on multiple measurement vectors (MMV) for rotating cone-shaped targets is proposed to realize Bi-RCI coherent processing and improve imaging performance. Based on the mixed mode signals, a MMV parametric model is established and measurement number coarse selection is proposed. Finally, a modified sparse Bayesian learning (MSBL) algorithm is introduced to reconstruct the target image. Simulation results demonstrate the validity and the superiority of the proposed method.
\end{abstract}

\section{Introduction}

Due to the separation of the transmitter and receiver, bistatic radar has better survivability and antijamming performance, can obtain more abundant target feature information, and can effectively reduce the system cost [1]. Therefore, a bistatic radar system has great advantages and potential in modern electronic warfare, and it has become a research hotspot of radar technology [2-6].

As one of the imaging techniques, the inverse synthetic aperture radar (ISAR) imaging using bistatic configuration can overcome some limitations of monostatic ISAR imaging and is exploited in territory air defense [7], ocean surveillance [8], and other fields. Currently, aiming at two features of the Bi-ISAR in terms of scaled image resolution and image shearing distortion, bistatic distortion correction methods and a crossrange scaling technique are studied [9-11]. With the continuous development of signal processing technology, bistatic three-dimensional (3-D) interferometric ISAR based on interference processing has gradually become a research hotspot [12-14]. Also, this kind of method can provide more abundant information. The methods above have played an important role in promoting the practical application of bistatic radar imaging systems. The Bi-ISAR imaging and the bistatic 3-D interferometric ISAR imaging are both ISAR-based imaging methods. Nevertheless, these methods are complicated and even ineffective for targets with internal motions due to the influence of target micromotion.

In recent years, radar coincidence imaging (RCI), which is one of the incoherent and computational imaging methods, has been proposed and studied by Li et al. [15]. Compared with ISAR imaging, RCI is highly effective for rapid real-time imaging and is suitable for static or quasistationary targets [16]. The ability of RCI processing to simplify the complicated motion compensation of a noncooperative target makes it applicable to both cooperative and noncooperative targets. There is a mode of the correlation between signals $\mathrm{A}$ and $\mathrm{B}$ in the $\mathrm{RCI}$ processing, where $\mathrm{A}$ is the 
detection signal and B is the received signal. Importantly, it is essential that signal A require time-space independence. In order to acquire the time-space independent signals, the core for achieving RCI is to design the transmitted signal. Consequently, a multitransmitting configuration with multiple independent subsources is reasonable for RCI and is used to produce the time-space independent signals. Compared with ISAR imaging, RCI does not rely on coherent focus processing to achieve the desired azimuth resolution, so imaging processing is much faster, which is an important superiority of RCI. Currently, a mixed mode radar coincidence imaging (MMRCI) system is proposed by Zhu et al. [17]. The transmitted signals of the MMRCI system consist of two parts: the coherent part used for the target detection and the incoherent part used for the target imaging. In this way, the image quality of the moving target can be improved by effectively reducing the target position estimation error with the coherent part of transmitted signals. Then, the incoherent part produces the time-space independent reference radiation field with high resolution but no target information. On the contrary, the echoes have no spatial resolution but carry the target information. Therefore, the RCI images can be acquired by processing the reference radiation field and the echoes together.

Based on the RCI principle, although bistatic RCI (BiRCI) has been introduced in our previous work [18], a linear imaging model is established to reconstruct images in this BiRCI. The linear imaging model is a single measurement vector (SMV) and has limited imaging performance. Currently, there are few researches on the RCI coherent processing method for rotating targets. Cone-shaped targets such as the missile are a kind of typical rotating targets, so taking the cone-shaped targets as the research object, Bi-RCI coherent processing is investigated in this paper. To realize $\mathrm{Bi}-\mathrm{RCI}$ coherent processing for rotating targets, two new problems need to be solved: one is how to exploit the echoes to conduct coherent accumulation and the other one is the determination of the coherent accumulation time to ensure the coherent processing performance. Hitherto, RCI uses a parametric model to reconstruct images. The advantage of this method is to improve the imaging resolution and to obtain super-resolution imaging results. Therefore, in this paper, in order to solve the first problem, the signal waveform is repeatedly transmitted to obtain coherent echoes for accumulation, and then a multiple measurement vector (MMV) parametric model is established to realize the Bi-RCI coherent accumulation process. For the other problem, due to the influence of target motion, the coherence accumulation time has an upper limit, which affects the measurement number of the MMV parametric model. By analyzing the Doppler and micro-Doppler effects of target motion and rotation, the constraints between coherent accumulation time and distance variation caused by target motion are derived, and the measurement number coarse selection of the MMV parametric model is finally obtained. The advantages of the proposed method can be summarized as follows: (1) because of the separation of the transceiver and receiver, the continuous waveform can be used to shorten the observation time to realize fast and real-time imaging of rotating targets; (2) the coherent accumulation time is short, so the influence of target motion and rotation can be neglected, and it is not necessary to implement the echo translational motion compensation in the imaging process; and (3) coherent processing can enhance the antinoise ability and further improve the imaging quality.

The paper is structured as follows. Section 2 introduces the signal model including transmitted signals and received signals and proposes a MMV parametric model and a measurement number coarse selection to realize Bi-RCI coherent processing. Then, the target image is reconstructed with a modified sparse Bayesian learning (MSBL) algorithm. Section 3 presents our simulation results to analyze the performance of the proposed method. In Section 4, we draw the conclusions of this paper.

\section{Bi-RCI Based on MMV}

2.1. Signal Model. In the Bi-RCI system shown in Figure 1, the radar coordinate system $O-X Y Z$ and the reference coordinate system $O^{\prime}-x y z$ are established. For $O-X Y Z$, the origin $O$ is located on the transmitter; the $X$-axis is selected along the base line $L$, connecting the transmitter and the receiver; and the $X O Y$ plane is determined by a rotating target, the transmitter, and the receiver together. The $Z$-axis is perpendicular to the $X O Y$ plane by the right-handed helix rule. For $O^{\prime}-x y z$, the origin $O^{\prime}$ is located on the rotating target, and $O^{\prime}-x y z$ is parallel to the radar coordinate system $O$ $X Y Z$. A uniform linear array (ULA) consisting of $N$ elements is used as the transmitting antenna. $N$ elements are distributed uniformly on the $X$-axis with equal interval $d$. The mixed mode signals of the hybrid configuration are transmitted by the ULA, comprising the coherent part and the incoherent part [17]. Assuming that the reference point $O^{\prime}$ is located at $\left(x_{0}, y_{0}, 0\right)$, the unit vectors along the Line-of-Sight (LOS) of the transmitter and receiver are $\mathbf{i}_{t}=\left[x_{0} / \sqrt{x_{0}^{2}+y_{0}^{2}}, y_{0} / \sqrt{x_{0}^{2}+y_{0}^{2}}, 0\right]^{T}$ and $\mathbf{i}_{r}=\left[\left(x_{0}-L\right) /\right.$ $\left.\sqrt{\left(x_{0}-L\right)^{2}+y_{0}^{2}}, y_{0} / \sqrt{\left(x_{0}-L\right)^{2}+y_{0}^{2}}, 0\right]^{T}$, respectively.

The coherent part of the mixed mode signals is used to analyze the distance variation and determine the measurement number. Because narrow-band signal makes the radar have high system sensitivity and large maximum detectable distance brought by small-signal bandwidth and noise coefficient [19], a single frequency narrowband (SFN) signal is used as this part. The signal is transmitted by the element located at origin $O$, expressed as

$$
s^{s f}(t)=\exp \left\{j 2 \pi\left(f_{c}+\Delta F\right) t\right\}
$$

where $f_{c}$ denotes the carrier frequency and $\Delta F$ denotes the frequency difference between the SFN signal frequency and the carrier frequency. The setting of $\Delta F$ needs to consider that the SFN signal can be separated from the mixed mode signals.

The incoherent part of the mixed mode signals is a random frequency modulation (RFM) signal, with the time-space independence. This signal can achieve imaging 


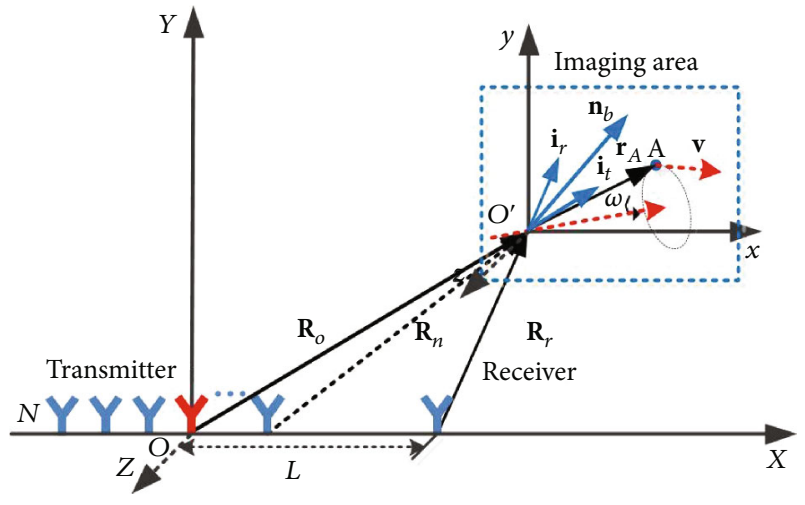

Figure 1: Bi-RCI system model.

for the rotating target. The signal transmitted by the $n$th element can be expressed as

$$
s_{n}^{\mathrm{in}}(t)=\operatorname{rect}\left(\frac{t}{T_{p}}\right) \exp \left\{j 2 \pi\left(f_{c}+C_{n}(t) \cdot B\right) t\right\},
$$

where $T_{p}$ is the pulse duration, $f_{c}$ is the carrier frequency that is the same as the coherent part, $B$ is the signal bandwidth, $C_{n}(t)$ are uniformly distributed in $[-0.5,0.5]$, and $n$ represents the element index that is $n=1,2, \cdots, N$. In addition,

$$
\operatorname{rect}(t)= \begin{cases}1, & 0<t \leq 1, \\ 0, & \text { else }\end{cases}
$$

Hence, the mixed mode signals is the summation of the coherent and incoherent signals, which can be described by

$$
s(t)=s^{s f}(t)+s^{\mathrm{in}}(t)=s^{s f}(t)+\sum_{n=1}^{N} s_{n}^{\mathrm{in}}(t)
$$

$A$ is a scatterer on the rotating target. The receiving antenna captures the echoes scattered from scatterer $A$, which can be expressed as

$$
y_{r}(t)=\sigma_{A} \cdot s\left(t-\tau_{A}\right),
$$

where $\sigma_{A}$ is the scattering coefficient of scatterer $A$ and $\tau_{A}$ is the time delay of the echoes scattered by scatterer $A$. Owing to the existence of the motion, time delay $\tau_{A}$ varies with time $t$. Moreover, the mixed mode signals are continuously transmitted during the radar observing period because of the separation of the transmitter and the receiver, and their time-frequency spectrum is shown in Figure 2.

2.2. MMV Parametric Model. Although the RFM signal and the SFN signal are mixed and transmitted together in the time domain, these two parts are mutually independent in the frequency domain. Hence, they can be distinguished in the frequency domain. Assume that the receiver can obtain synchronous signals with good performance to achieve time and frequency synchronization and that the translational motion and rotation within one pulse duration $T_{p}$ are ignored. After using a band-pass filter, the incoherent part of the echoes can be expressed as

$$
\begin{aligned}
y_{r}^{\text {in }}\left(\hat{t}, t_{m}\right)= & \sigma_{A} \cdot \sum_{n=1}^{N} \operatorname{rect}\left(\frac{\hat{t}-R_{A}^{n}\left(t_{m}\right) / c}{T_{p}}\right) \\
& \cdot \exp \left\{j 2 \pi\left(f_{c}+C_{n}(\widehat{t}) \cdot B\right)\left(\hat{t}-\frac{R_{A}^{n}\left(t_{m}\right)}{c}\right)\right\},
\end{aligned}
$$

where $t=\widehat{t}+t_{m}=\widehat{t}+(m-1) T_{p}$ and $\widehat{t}$ and $t_{m}$ are the fasttime variate and slow-time variate, respectively. $c$ is the speed of light in space. $R_{A}^{n}\left(t_{m}\right)$ denotes the time-varying distance that the echoes travel from the $n$th element to the receiving antenna and can be expressed as

$R_{A}^{n}\left(t_{m}\right)=\left\|\mathbf{R}_{n}+\boldsymbol{v} t_{m}+\mathbf{R}_{\mathrm{rot}}\left(t_{m}\right) \mathbf{r}_{A}\right\|+\left\|\mathbf{R}_{r}+\mathbf{v} t_{m}+\mathbf{R}_{\mathrm{rot}}\left(t_{m}\right) \mathbf{r}_{A}\right\|$,

where $\mathbf{R}_{n}$ denotes the vector from the $n$th element to $O^{\prime}, \mathbf{R}_{r}$ denotes the vector from the receiving antenna to $O^{\prime}, \mathbf{v}$ is the target velocity, $\mathbf{R}_{\text {rot }}\left(t_{m}\right)$ denotes a 3-D rotating matrix, and $\mathbf{r}_{A}=\left[x_{A}, y_{A}, z_{A}\right]^{T}$ is the position vector of $A$ in the reference coordinate system. Assuming that the target is located on a plane $I$, and the variation in the phase distribution caused by the translational motion and rotation is ignored, $y_{r}^{\text {in }}\left(\widehat{t}, t_{m}\right)$ can be expressed as the superposition of reference signal $S\left(\widehat{t}, t_{m}, \mathbf{r}\right)[15]$ :

$$
y_{r}^{\text {in }}\left(\widehat{t}, t_{m}\right)=\int_{I} \sigma_{r} \cdot S\left(\widehat{t}, t_{m}, \mathbf{r}\right) d \mathbf{r},
$$

where

$$
\begin{aligned}
S\left(\widehat{t}, t_{m}, \mathbf{r}\right)= & \sum_{n=1}^{N} \operatorname{rect}\left(\frac{\hat{t}-R^{n}\left(t_{m}, \mathbf{r}\right) / c}{T_{p}}\right) \\
& \cdot \exp \left\{j 2 \pi\left(f_{c}+C_{n}(\widehat{t}) \cdot B\right)\left(\hat{t}-\frac{R^{n}\left(t_{m}, \mathbf{r}\right)}{c}\right)\right\},
\end{aligned}
$$

$R^{n}\left(t_{m}, \mathbf{r}\right)=\left\|\mathbf{R}_{n}+\mathbf{v} t_{m}+\mathbf{R}_{\text {rot }}\left(t_{m}\right) \mathbf{r}\right\|+\left\|\mathbf{R}_{r}+\mathbf{v} t_{m}+\mathbf{R}_{\text {rot }}\left(t_{m}\right) \mathbf{r}\right\|$.

After the discretization of the fast-time domain, the incoherent part of the echoes can be expressed as $\mathbf{y}\left(t_{m}\right)=$ $\left[y_{1}\left(t_{m}\right), y_{2}\left(t_{m}\right), \cdots, y_{P}\left(t_{m}\right)\right]^{T} \in \mathbb{C}^{P \times 1}$, in which

$$
y_{p}\left(t_{m}\right)=y_{r}^{\text {in }}\left(\widehat{t}_{p}, t_{m}\right)=\sigma_{A} \cdot \sum_{n=1}^{N} \exp \left\{j 2 \pi f_{p}\left(\widehat{t}_{p}-\frac{R_{A}^{n}\left(t_{m}\right)}{c}\right)\right\},
$$




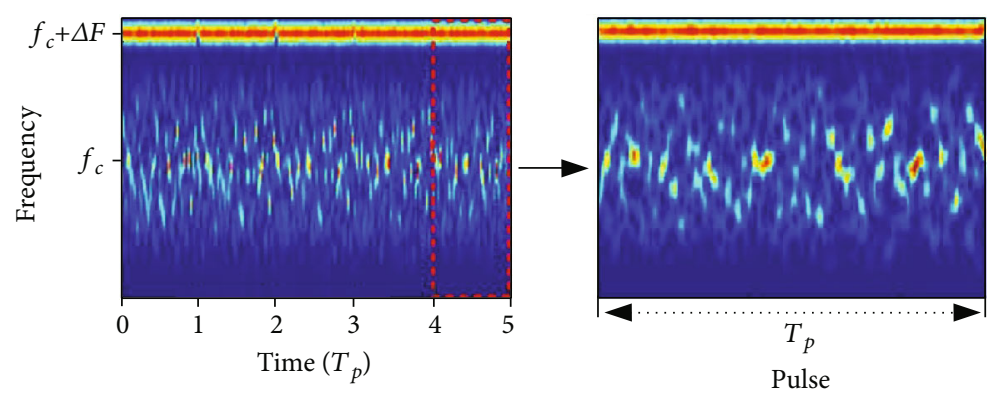

FIGURE 2: The time-frequency spectrum of the mixed mode signals.

where $\widehat{t}_{p}$ is the $p$ th sampling time, $f_{p}=\left(f_{c}+C_{n}\left(\widehat{t}_{p}\right) \cdot B\right)$ denotes the signal frequency at this sampling time $\hat{t}_{p}$, and $P$ is the sample number in the fast-time domain.

According to the RCI theory, the parameterized method can be used to establish a parametric model, and the superresolution image can be constructed by solving this problem [20]. The imaging area is first discretized as a grid of $Q$ cells. The position vectors of the cells are expressed as $V=\left\{\mathbf{r}_{q} \mid q\right.$ $=1,2, \cdots, Q\}$, where $\mathbf{r}_{q}=\left[x_{q}, y_{q}, z_{q}\right]^{T}$ is the position vector of the $q$ cell in the reference coordinate system $O^{\prime}-x y z$. The scattering coefficients of these cells form a vector $\sigma\left(t_{m}\right)=$ $\left[\sigma_{1}\left(t_{m}\right), \sigma_{2}\left(t_{m}\right), \cdots, \sigma_{Q}\left(t_{m}\right)\right]^{T} \in \mathbb{C}^{Q \times 1}$. Then, after sampling in the fast-time domain, the reference signal vector of the imaging area at $\widehat{t}_{p}$ can be calculated:

$$
\mathbf{S}_{p}\left(t_{m}\right)=\left[S\left(\hat{t}_{p}, t_{m}, \mathbf{r}_{1}\right), S\left(\hat{t}_{p}, t_{m}, \mathbf{r}_{2}\right), \cdots, S\left(\hat{t}_{p}, t_{m}, \mathbf{r}_{Q}\right)\right] .
$$

Hence, the measurement matrix formed by the reference signal vectors can be written as

$$
\begin{aligned}
\mathbf{S}\left(t_{m}\right) & =\left[\mathbf{S}_{1}\left(t_{m}\right), \mathbf{S}_{2}\left(t_{m}\right), \cdots, \mathbf{S}_{P}\left(t_{m}\right)\right]^{T} \\
& =\left[\begin{array}{cccc}
S\left(\widehat{t}_{1}, t_{m}, \mathbf{r}_{1}\right) & S\left(\widehat{t}_{1}, t_{m}, \mathbf{r}_{2}\right) & \cdots & S\left(\widehat{t}_{1}, t_{m}, \mathbf{r}_{Q}\right) \\
S\left(\widehat{t}_{2}, t_{m}, \mathbf{r}_{1}\right) & S\left(\widehat{t}_{2}, t_{m}, \mathbf{r}_{2}\right) & \cdots & S\left(\widehat{t}_{2}, t_{m}, \mathbf{r}_{Q}\right) \\
\vdots & \vdots & \ddots & \vdots \\
s\left(\widehat{t}_{P}, t_{m}, \mathbf{r}_{1}\right) & S\left(\widehat{t}_{P}, t_{m}, \mathbf{r}_{2}\right) & \cdots & S\left(\widehat{t}_{P}, t_{m}, \mathbf{r}_{Q}\right)
\end{array}\right] .
\end{aligned}
$$

Finally, we consider the influences of noise. Based on the parameterized method, the equation is expressed as

$$
\mathbf{y}\left(t_{m}\right)=\mathbf{S}\left(t_{m}\right) \cdot \sigma\left(t_{m}\right)+\mathbf{e}\left(t_{m}\right)
$$

where $\mathbf{e}\left(t_{m}\right) \in \mathbb{C}^{P \times 1}$ is a noise vector at $t_{m}$. When observation time is $T_{p}$, the parametric model is established based on a single measurement vector (SMV), expressed as formula (14). If $M$ pulses are transmitted in the coherent accumulation time $T_{M}$, echo matrix $\mathbf{Y}$ can be obtained and expressed as $\mathbf{Y}=\left[\mathbf{y}\left(t_{1}\right), \mathbf{y}\left(t_{2}\right), \cdots, \mathbf{y}\left(t_{M}\right)\right] \in \mathbb{C}^{P \times M}$, where $\mathbf{y}\left(t_{m}\right) \in \mathbb{C}^{P \times 1}, m$ $=1,2, \cdots, M$ is the echo vector of the $m$ th pulse.
According to (9), (10), and (13), the measurement matrix $\boldsymbol{S}\left(t_{m}\right)$ varies with $t_{m}$ because of the target motions. To establish a parametric model based on multiple measurement vectors (MMV), a measurement matrix not varying with $t_{m}$ is necessary. It is feasible to ignore the distance variation brought by the target translational motion and rotation in short coherent accumulation time $T_{M}$, because the target is still located in the grid discretized initially at $t_{1}$. Under this condition, a MMV parametric model can be obtained and expressed as

$$
\mathbf{Y}=\mathbf{S} \cdot \Sigma+\mathbf{E},
$$

where the measurement matrix $\mathbf{S}=\mathbf{S}\left(t_{1}\right), \mathbf{E}$ is a noise matrix, and $\mathbf{E}=\left[\mathbf{e}\left(t_{1}\right), \mathbf{e}\left(t_{2}\right), \cdots, \mathbf{e}\left(t_{M}\right)\right] \in \mathbb{C}^{P \times M} . \Sigma=\left[\sigma\left(t_{1}\right), \sigma\left(t_{2}\right), \cdots, \sigma\right.$ $\left.\left(t_{M}\right)\right] \in \mathbb{C}^{\mathrm{Q} \times M}$ is a scattering coefficient matrix, and $\left\{\sigma\left(t_{m}\right) \mid\right.$ $m=1,2, \cdots, M\}$ has the same sparse structure in $T_{M}$ under the condition of an ideal scatterer model. Hence, the determination of the observation time is significant to establish the MMV parametric model. The coherent accumulation time decides the measurement number of the model; in other words, we need determine a suitable measurement number, which is helpful to coherent processing of Bi-RCI.

2.3. Measurement Number Coarse Selection. By filtering, the coherent part of the echoes can be denoted

$$
y_{r}^{s f}(t)=\sigma_{A} \cdot \exp \left\{j 2 \pi f_{s}\left(t-\frac{R_{A}(t)}{c}\right)\right\},
$$

where $f_{s}=f_{c}+\Delta F$ denotes the frequency of the SFN signal, and $R_{A}(t)$ denotes the time-varying distance that the echoes travel, which is expressed as

$$
R_{A}(t)=\left\|\mathbf{R}_{o}+\mathbf{v} t+\mathbf{R}_{\mathrm{rot}}(t) \mathbf{r}_{A}\right\|+\left\|\mathbf{R}_{r}+\mathbf{v} t+\mathbf{R}_{\mathrm{rot}}(t) \mathbf{r}_{A}\right\|,
$$

where $\mathbf{R}_{o}$ denotes the vector from the first element of the ULA to $O^{\prime}$ and $\mathbf{R}_{\text {rot }}(t)$ denotes a 3-D rotating matrix. By taking the time derivative of the phase $\Phi^{s f}(t)=2 \pi f_{s}(t-$ $\left.R_{A}(t) / c\right)$, the Doppler frequency shift of $y_{r}^{s f}(t)$ is derived as follows [21]: 
$f_{d}=\frac{1}{2 \pi} \frac{d}{d t} \Phi^{s f}(t)=-\frac{f_{s}}{c} \frac{d}{d t} R_{A}(t)=-\frac{f_{s}}{c}\left[\mathbf{v}+\frac{d}{d t}\left(\mathbf{R}_{\mathrm{rot}}(t) \mathbf{r}_{A}\right)\right]^{T} \mathbf{n}_{b}(t)$

where $\mathbf{n}_{b}(t)$ is a direction vector of the bistatic angle bisector at $t$. The first term of (18) is the Doppler shift caused by the target translational motion, while the second term denotes the micro-Doppler (m-D) frequency modulation induced by the rotation of scatterer $A$. The distance variation rate of scatterer $A$ can be obtained:

$$
\frac{d}{d t} R_{A}(t)=-\frac{c}{f_{s}} f_{d}=\left[\mathbf{v}+\frac{d}{d t}\left(\mathbf{R}_{\mathrm{rot}}(t) \mathbf{r}_{A}\right)\right]^{T} \mathbf{n}_{b}(t) .
$$

Assuming that the rotating angular velocity is $\omega=$ $\left[\omega_{x}, \omega_{y}, \omega_{z}\right]^{T}, \quad \Omega=\|\omega\|$, and $\omega^{\prime}=\omega / \Omega=\left[\omega_{x}^{\prime}, \omega_{y}^{\prime}, \omega_{z}^{\prime}\right]^{T}$, the skew symmetric matrix generated by $\omega^{\prime}$ can be expressed as

$$
\widehat{\omega}^{\prime}=\left[\begin{array}{ccc}
0 & -\omega_{z}^{\prime} & \omega_{y}^{\prime} \\
\omega_{z}^{\prime} & 0 & -\omega_{x}^{\prime} \\
-\omega_{y}^{\prime} & \omega_{x}^{\prime} & 0
\end{array}\right],
$$

and the rotating matrix $\mathbf{R}_{\mathrm{rot}}(t)$ is determined by

$$
\mathbf{R}_{\mathrm{rot}}(t)=\exp \left(\Omega \widehat{\omega}^{\prime} t\right)=I+\widehat{\omega}^{\prime} \sin \Omega t+\widehat{\omega}^{\prime 2}(1-\cos \Omega t) .
$$

The $\mathrm{m}-\mathrm{D}$ frequency can be described by

$$
f_{m-D}=-\frac{\Omega f_{s}}{c}\left[\widehat{\omega}^{\prime}\left(\widehat{\omega}^{\prime} \sin \Omega t+\mathbf{I} \cos \Omega t\right) \mathbf{r}_{A}\right]^{T} \mathbf{n}_{b}(t)
$$

By the Taylor Formula, the expansion of (22) can be obtained:

$$
\begin{aligned}
f_{m-D}= & -\frac{\Omega f_{s}}{c}\left[\widehat { \omega } ^ { \prime } \left(\widehat{\omega}^{\prime}(\Omega t+o(t))\right.\right. \\
& \left.\left.+\mathbf{I}\left(1-\frac{1}{2}(\Omega t)^{2}+o\left(t^{2}\right)\right)\right) \mathbf{r}_{A}\right]^{T} \mathbf{n}_{b}(t)
\end{aligned}
$$

When $t \longrightarrow 0$,

$$
f_{m-D} \approx-\frac{\Omega f_{s}}{c}\left[\widehat{\omega}^{\prime}\left(\widehat{\omega}^{\prime} \Omega t+\mathbf{I}\right) \mathbf{r}_{A}\right]^{T} \mathbf{n}_{b}(t) .
$$

Though $\mathbf{n}_{b}(t)$ is time-varying, its effect on $f_{d}$ is negligible in a short time. Therefore, $\mathbf{n}_{b}(t) \approx \mathbf{n}_{b 0}$, where $\mathbf{n}_{b 0}$ is the direction vector of a bistatic angle bisector at the initial position. Then, the distance variation rate can be written as $\frac{d}{d t} R_{A}(t)=\mathbf{v}^{T} \mathbf{n}_{b}(t)-\frac{c}{f_{s}} f_{m-D} \approx \mathbf{v}^{T} \mathbf{n}_{b 0}+\Omega\left[\widehat{\omega}^{\prime}\left(\widehat{\omega}^{\prime} \Omega t+\mathbf{I}\right) \mathbf{r}_{A}\right]^{T} \mathbf{n}_{b 0}$.

In $T_{M}$, to make $\mathbf{S}\left(t_{1}\right) \approx \mathbf{S}\left(t_{m}\right), m=1,2, \cdots, M$, the inequation needs to be satisfied as follows:

$$
\begin{aligned}
\left|\int_{0}^{T_{M}} \frac{d}{d t} R_{A}(t) d t\right| & =\left|\int_{0}^{T_{M}}\left(\mathbf{v}^{T} \mathbf{n}_{b 0}+\Omega\left[\widehat{\omega}^{\prime}\left(\widehat{\omega}^{\prime} \Omega t+\mathbf{I}\right) \mathbf{r}_{A}\right]^{T} \mathbf{n}_{b 0}\right) d t\right| \\
& <\frac{\delta_{r}}{2}
\end{aligned}
$$

where $\delta_{r}$ denotes the cell size of the grid. If $T_{M}$ could be settled, the measurement number can be determined by $M=$ $\left\lfloor T_{M} / T_{p}\right\rfloor$, in which $\lfloor *\rfloor$ denotes downward integer operation. By conducting the $\mathrm{TF}$ analysis and exploiting the existing methods [22], $\mathbf{v}$ and $\Omega$ can be obtained easily, while $\omega \wedge^{\prime}$ is more difficult to acquire. This causes $T_{M}$ to not settle. Considering the prior information of the imaging target such as the values of the translational motion velocity, the rotating radius, and the rotating angular velocity, the values of $\Omega$ and $\mathbf{r}_{A}$ are both much smaller than that of $\mathbf{v}$, which make $\Omega \widehat{\omega}^{\prime} \mathbf{r}_{A} \ll|\mathbf{v}|$ work. Hence, the inequation can be expressed as

$$
\left|\int_{0}^{T_{M}}\left(\mathbf{v}^{T} \mathbf{n}_{b 0}\right) d t\right|<\frac{\xi \delta_{r}}{2}
$$

where $\xi$ denotes an attenuation factor and $\xi \in(0,1)$. The function of the attenuation factor is to constrain the upper bound of distance variation, considering that the distance variation caused by the target rotation is ignored in the analysis process. If the value of $\xi$ is small, the MMV parametric model established based on the obtained measurement number is more likely to succeed, but the imaging performance may not be significantly improved. This is because the coherent accumulation time is too short. On the contrary, if the value of $\xi$ is larger, the risk of imaging failure will increase. In this paper, we mainly study how to achieve Bi-RCI coherent processing to improve imaging performance. Therefore, in order to ensure the reliability of establishing a MMV parametric model, we select a small attenuation factor.

2.4. Image Reconstruction. The MMV parametric model expressed as (15) can be solved by dealing with the following optimization problem:

$$
\begin{array}{ll}
\min & \frac{1}{M} \sum_{m=1}^{M}\left\|\sigma\left(t_{m}\right)\right\|_{0}, \\
\text { s.t. } & \|\mathbf{Y}-\mathbf{S} \cdot \Sigma\|_{F}<\varepsilon .
\end{array}
$$

Considering both the sparse feature and the prior information of the signal and consulting the algorithm of [23], we propose the MSBL algorithm to reconstruct the image 


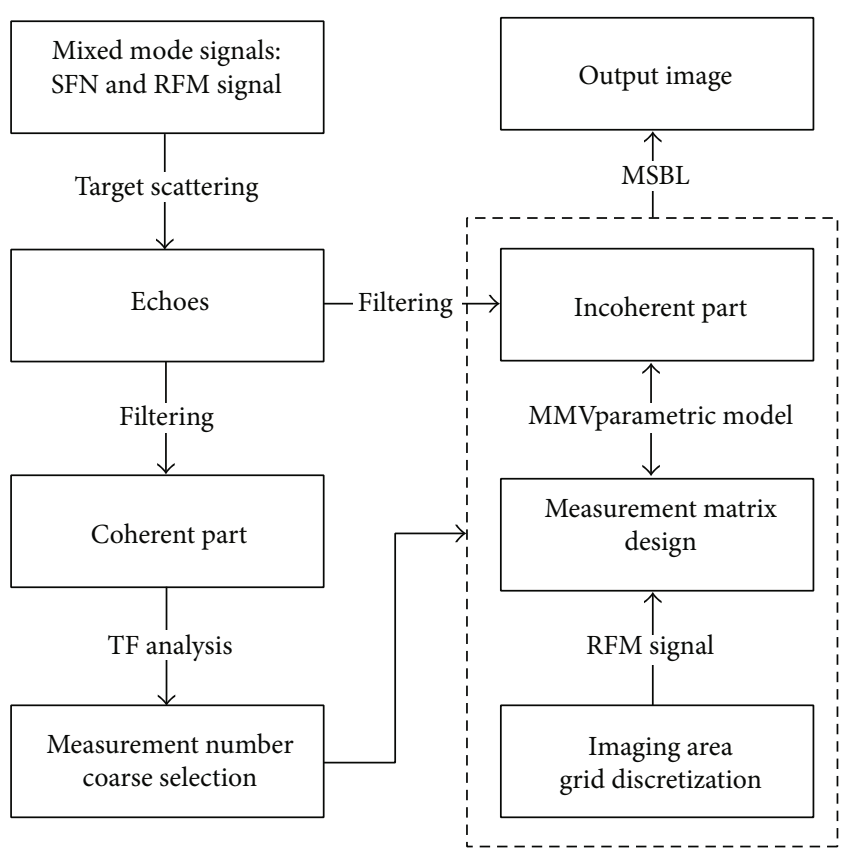

FIGURE 3: The flowchart of Bi-RCI based on the MMV method.

by deriving the sparse Bayesian formulation and conducting the Bayesian inference.

For the noise $\Sigma$, assuming that it is the white complex Gaussian noises, we have

$$
p\left(\mathbf{E} \mid \alpha_{0}\right)=\prod_{m=1}^{M} C N\left(\mathbf{e}\left(t_{m}\right) \mid \mathbf{0}, \alpha_{0}^{-1} \mathbf{I}\right)
$$

where $\alpha_{0}=\sigma^{-2}$ is the noise precision and $\sigma^{2}$ denotes the noise variance. For $\alpha_{0}$, a Gamma hyperprior is assumed because it is a conjugate prior of the Gaussian distribution, and it is given by

$$
p\left(\alpha_{0} ; a, b\right)=\Gamma\left(\alpha_{0} \mid a, b\right)
$$

where $\Gamma\left(\alpha_{0} \mid a, b\right)=[\Gamma(a)]^{-1} b^{a} \alpha_{0}^{a-1} e^{-b \alpha_{0}}, \Gamma\left(v_{1}\right)$ is the Gamma function, and $a$ and $b$ are constants with $a, b \longrightarrow 0$.

For the scattering coefficient matrix $\Sigma$, a two-layer Gaussian-Gamma hierarchical prior is adopted: $p(\Sigma ; \rho)=$ $\int p(\Sigma \mid \alpha) p(\alpha ; \rho) d \alpha$, in which

$$
\begin{gathered}
p(\Sigma \mid \alpha)=\prod_{m=1}^{M} C N\left(\sigma\left(t_{m}\right) \mid 0, \Lambda\right), \\
p(\alpha ; \rho)=\prod_{q=1}^{Q} \Gamma\left(\alpha_{q} \mid 1, \rho\right),
\end{gathered}
$$

where $\rho>0, \alpha \in \mathbb{R}^{Q}$, and $\Lambda=\operatorname{diag}(\alpha)$.

Then, Bayesian inference is conducted, and we treat $\Sigma$ as a hidden variable and $\alpha_{0}$ and $\alpha$ as hyperparameters. Here, the expectation-maximization algorithm is implemented as the Bayesian inference principle. It is easy to obtain that the pos-
TABLE 1: The parameters of transmitted signals.

\begin{tabular}{lc}
\hline Parameter & Value \\
\hline Carrier frequency, $f_{c}$ & $10 \mathrm{GHz}$ \\
Bandwidth, $B$ & $2 \mathrm{GHz}$ \\
Pulse duration, $T_{p}$ & $2 \mu \mathrm{s}$ \\
Frequency difference, $\Delta F$ & $1.5 \mathrm{GHz}$ \\
\hline
\end{tabular}

terior distribution of $\Sigma$ follows a Gaussian distribution, whose probability density function (PDF) is

$$
p\left(\Sigma \mid \mathbf{Y}, \alpha_{0}, \alpha\right)=\prod_{m=1}^{M} C N\left(\sigma\left(t_{m}\right) \mid \mu\left(t_{m}\right), \Phi\right)
$$

with

$$
\begin{gathered}
\mu\left(t_{m}\right)=\alpha_{0} \Phi \mathbf{S}_{d}{ }^{H} \mathbf{y}\left(t_{m}\right), \\
\Phi=\left(\alpha_{0} \mathbf{S}^{H} \mathbf{S}+\Lambda^{-1}\right)^{-1} .
\end{gathered}
$$

For the acquisition of $\mu\left(t_{m}\right), m=1,2, \cdots, M$ and $\Phi$, the estimates of the hyperparameters $\alpha_{0}$ and $\alpha$ are needed. Denoting $\mathbf{U}=\left[\mu\left(t_{1}\right), \mu\left(t_{2}\right), \cdots, \mu\left(t_{M}\right)\right]$ and following similar procedures as in [23], we can acquire the updates of $\alpha_{0}$ and $\alpha$ as follows:

$$
\alpha_{0}^{\text {new }}=\frac{M P+a-1}{E\left\{\|\mathbf{Y}-\mathbf{S} \cdot \Sigma\|_{F}^{2}\right\}+b},
$$

$$
\alpha_{q}^{\text {new }}=\frac{\sqrt{1+4 \rho E\left\{\left\|\Sigma^{q}\right\|_{2}^{2}\right\}}-1}{2 \rho}, \quad q=1,2, \cdots, Q,
$$

where $E\left\{\|\mathbf{Y}-\mathbf{S} \cdot \Sigma\|_{F}^{2}\right\}=\|\mathbf{Y}-\mathbf{S} \cdot \mathbf{U}\|_{F}^{2}+\alpha_{0}^{-1} \sum_{q=1}^{Q} \gamma_{q}$ with $\gamma_{q}$ $=1-\alpha_{q}^{-1} \Phi_{q q}, E\left\{\left\|\Sigma^{q}\right\|_{2}^{2}\right\}=\left\|\mathbf{U}^{q}\right\|_{2}^{2}+\Phi_{q q} . \mathbf{U}^{q}$ denotes the $q$ th row vector of $\mathbf{U}$, and $\Phi_{q q}$ denotes the $q$ th row and the $q$ th column element of $\Phi$. $\|\cdot\|_{2}$ and $\|\cdot\|_{F}$ denote the $l_{2}$ norm and the Frobenius norm, respectively.

The MSBL algorithm is implemented as follows. The hyperparameters $\alpha_{0}$ and $\alpha$ are initialized. After that, $\mu\left(t_{m}\right)$, $m=1,2, \cdots, M$, and $\Phi$ can be calculated using the current values of the hyperparameters according to (34) and (35), respectively. Then, the hyperparameters $\alpha_{0}$ and $\alpha$ are updated according to (36) and (37), respectively. The steps above are repeated, and when the convergence criterion is satisfied, reaching the maximum number of iterations or $\left\|\alpha^{i+1}-\alpha^{i}\right\|_{2} /\left\|\alpha^{i}\right\|_{2}<\beta$, the iteration is ended. The reconstruction scattering coefficient vector $\widehat{\sigma}=(1 / M) \sum_{m=1}^{M} \mid$ $\mu\left(t_{m}\right) \mid$.

In summary, the specific steps of the proposed method are given as follows:

Step 1. By filtering, obtain the coherence of the echoes and conduct TF analysis. According to the constraints between the 


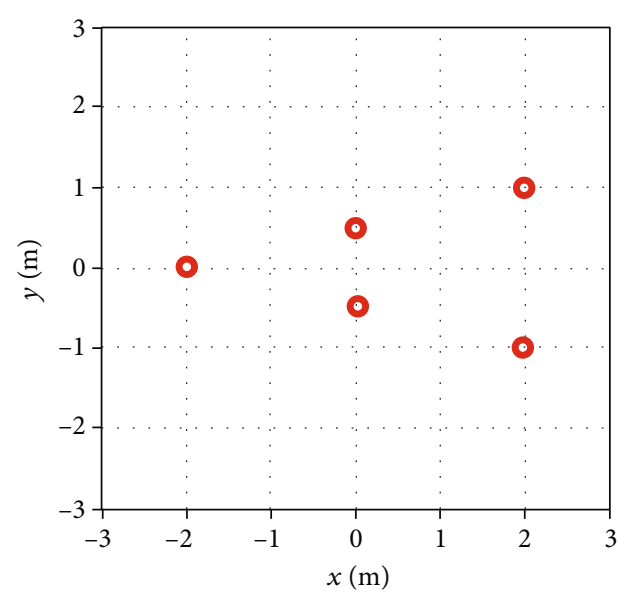

(a)

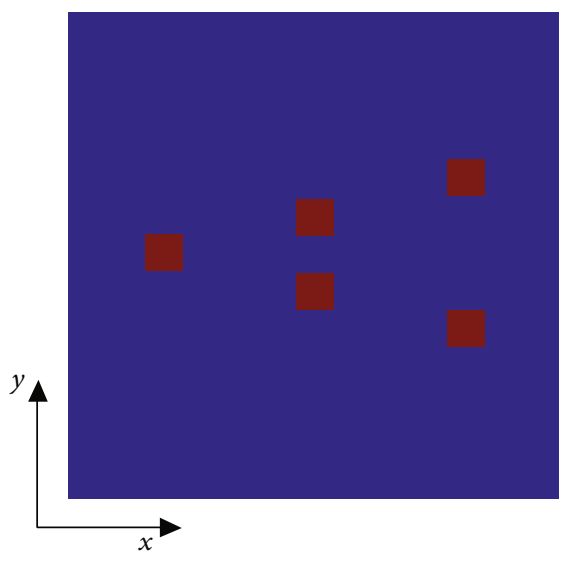

(b)

Figure 4: Target model: (a) scatterer position distribution; (b) scattering coefficient.

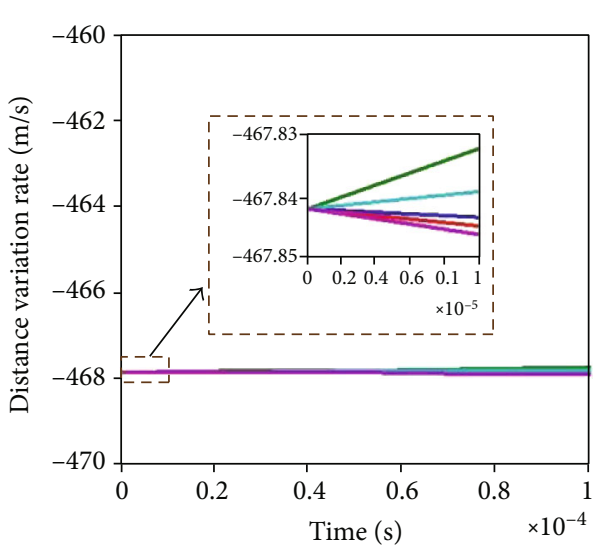

(a)

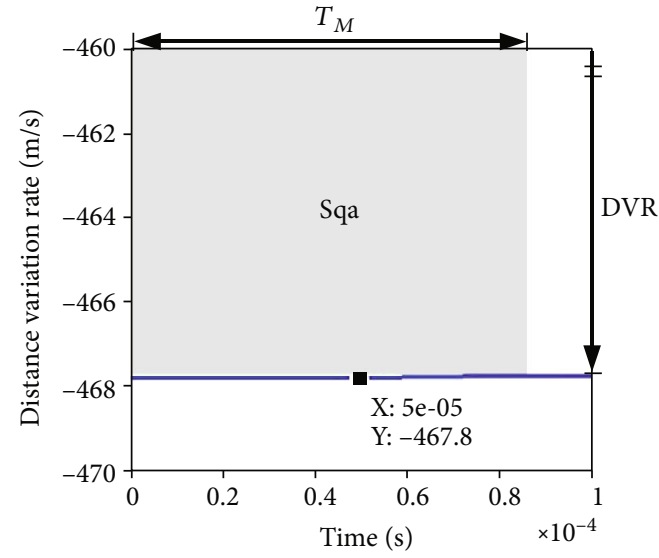

(b)

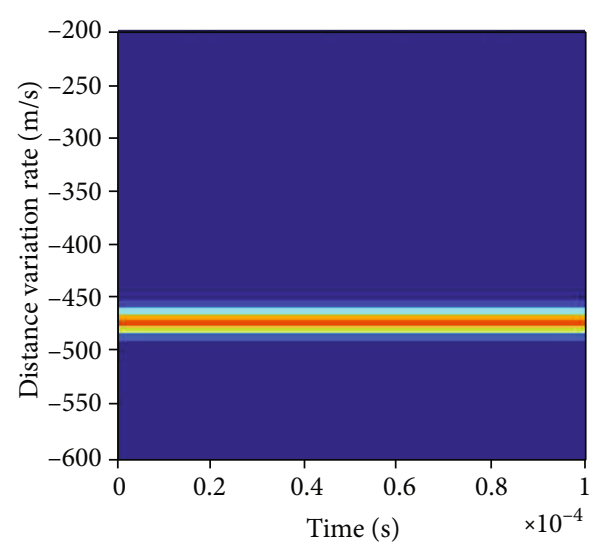

(c)

FIgURE 5: The distance variation rate with time variation. (a) The theoretical curves of the target with 5 scatterers. (b) The overall curve of distance variation rate obtained by the method of phase difference. (c) The overall curve of distance variation rate obtained by the method of Gabor Transform.

coherent accumulation time and the distance variation, calculate the measurement number.

Step 2. According to the prior information such as the target position and rough target size, determine the refer- ence point and imaging area and discretize the imaging area as a grid.

Step 3. Calculate the reference signal vectors in the imaging area and design the measurement matrix. 


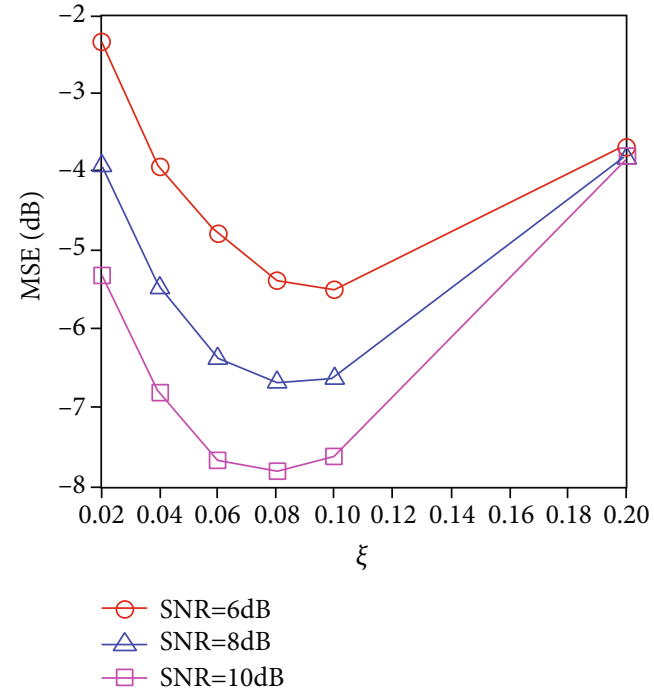

FIGURE 6: Imaging performance with attenuation factor varying under different SNR conditions.

Step 4. By filtering, obtain the incoherence of the echoes and establish the MMV parametric model.

Step 5. Solve the MMV parametric model and reconstruct the target image with the MSBL algorithm.

The flowchart of Bi-RCI based on MMV is depicted in Figure 3.

\section{Simulation and Analysis}

This part performs simulation experiments to validate the proposed method. The element number of the ULA is $N$ $=12$, and all of the elements are set in $[0 \mathrm{~m}, 11 \mathrm{~m}]$ of the $X$-axis with an interval of $1 \mathrm{~m}$. The baseline length $L$ $=50 \mathrm{~km}$, and the receiving antenna is located at $(50 \mathrm{~km}$, $0 \mathrm{~km}$, and $0 \mathrm{~km}$ ). The first element of the ULA transmits the mixed mode signals including the SFM signal and the RFM signal, and the others only transmit the RFM signal. The key simulation parameters of transmitted signals are chosen and listed in Table 1. For the coherent part of echoes, the sample number is 40000 in $1 \mathrm{~s}$. For the incoherent part of the echoes, the sample number in the fast-time domain is $P=256$ in $T_{p}$.

We assume a cone-shaped target as shown in Figure 4. The reference point is located at $(55 \mathrm{~km}, 20 \mathrm{~km}$, and $0 \mathrm{~km})$. The target moves with a velocity $\mathbf{v}=[-281.9380 \mathrm{~m} / \mathrm{s}$, $-102.5229 \mathrm{~m} / \mathrm{s}, 0 \mathrm{~m} / \mathrm{s}]^{T}$ and rotates with a rotating angular velocity $\omega=[-25.3911 \mathrm{rad} / \mathrm{s},-9.2331 \mathrm{rad} / \mathrm{s}, 0 \mathrm{rad} / \mathrm{s}]^{T}$. In the simulation experiment, the imaging area of $6 \mathrm{~m} \times 6 \mathrm{~m}$ was discretized to a grid of $13 \times 13$ cells, with a range resolution of $\delta_{r}=0.5 \mathrm{~m}$. The target scatterers are located at the cell centers.

We verify the measurement number coarse selection and introduce the specific implementation process. By filtering the echoes to acquire the coherent part and conducting the $\mathrm{TF}$ analysis, the curve of the distance variation rate with time

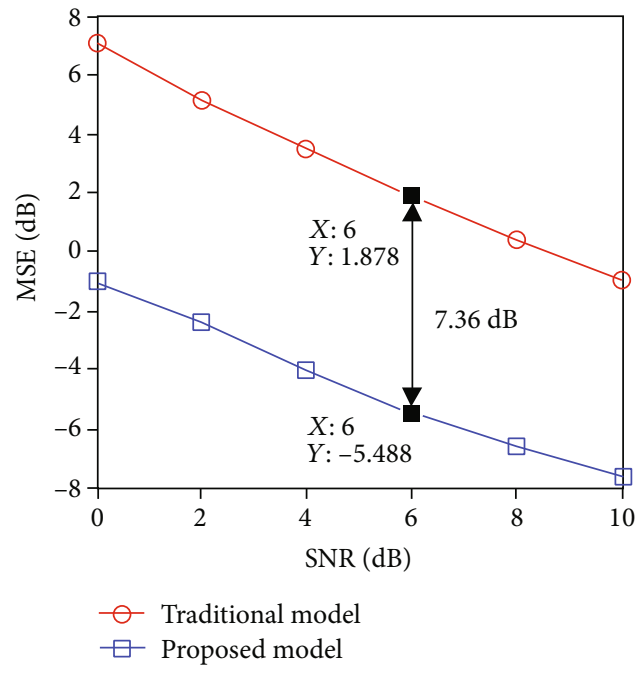

FIGURE 7: Imaging performance of the traditional and proposed models as a function of the SNR.

variation is obtained, as shown in Figure 5(a). It shows the theoretical curves of the distance variation rate of all scatterers. Theoretically, due to the existence of rotation of different scatterers, the distance variation rate of each scatterer is slightly different. However, the difference of the distance variation rate among different scatterers is very small compared with the overall distance variation rate. Depicted as Figures 5(b) and 5(c), the target distance variation rate obtained by the method of phase difference or Gabor Transform is a straight line, which is a constant, and the modulus is expressed by DVR. The distance variation rate is mainly caused by the target motion. This is mainly because the resolution of the above-mentioned method cannot meet the requirements of resolving the difference between the distance variation rates of different scatterers. Here, the symbol of the distance variation rate indicates the increase or decrease of the target's distance from the radar, and the minus sign indicates that the target is getting closer and closer to the radar, and the distance decreases. As shown in Figure 5(b), the area $S q a$ represents the increment of distance variation, that is, $S$ $q a \approx\left|\int_{0}^{T_{M}}\left(\mathbf{v}^{T} \mathbf{n}_{b 0}\right) d t\right|=D V R \cdot T_{M}$. Finally, under the condition of satisfying the inequation (27), the maximum of $T_{M}$ is calculated and obtained.

Then, the influence of the attenuation factor on the performance of the MMV parametric model is verified, and the curves are obtained as shown in Figure 6. In MSBL, we set $\rho=0.01$ and $a=b=10^{-4}$. We initialize $\alpha_{0}$ $=100 M / \sum_{m=1}^{M} \operatorname{var}(\mathbf{Y})$ and $\alpha=(1 / M) \sum_{m=1}^{M}\left|\mathbf{S}^{H} \mathbf{Y}\right|$. We set $\beta$ $=10^{-3}$ and the maximum number of iterations to 500 . To act as the reconstruction performance metric, the mean square error (MSE) is defined as

$$
M S E=10 \log \left(\frac{1}{K} \sum_{k=1}^{K} \frac{\|\widehat{\sigma}-\tilde{\sigma}\|_{2}^{2}}{\|\tilde{\sigma}\|_{2}^{2}}\right),
$$




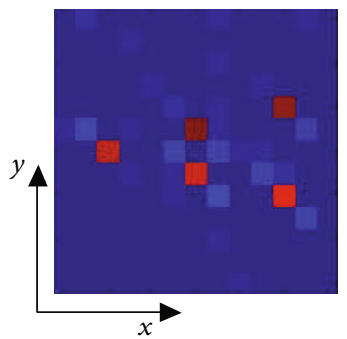

(a)

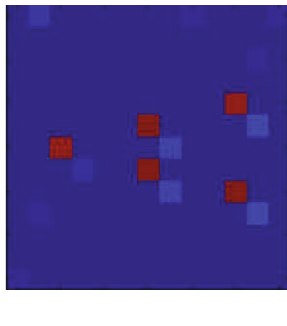

(b)

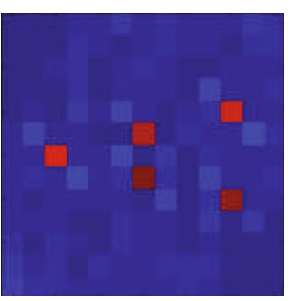

(c)

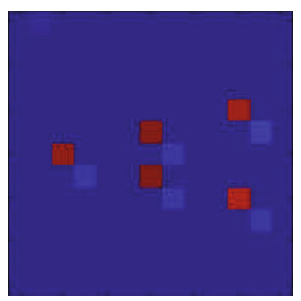

(d)

FIGURE 8: Imaging results using different models and different reconstruction algorithms under the condition of SNR $=10 \mathrm{~dB}$. (a) Using the traditional model and the OMP algorithm, MSE $=-0.93 \mathrm{~dB}$. (b) Using the proposed model and the MMV-OMP algorithm, MSE $=-5.12 \mathrm{~dB}$. (c) Using the traditional model and the MSBL algorithm, MSE $=-0.95 \mathrm{~dB}$. (d) Using the proposed model and the MSBL algorithm, MSE $=-7.60 \mathrm{~dB}$.

where $K$ is the number of Monte Carlo trials, and $K=30$ in this paper. $\widehat{\sigma}$ is the estimated scattering coefficient vector, and $\tilde{\sigma}$ is the true scattering coefficient vector. Figure 6 shows the MSE with the attenuation factor varying under different SNR conditions. It can be seen that each curve is a single peak curve, which indicates that there is an optimal attenuation factor, so that the MMV parametric model can obtain the optimal imaging results. The coherent accumulation time is proportional to the attenuation factor. Therefore, when the attenuation factor is less than the optimal attenuation factor, the imaging results of the MMV parametric model will get better and better with the increase of the coherent accumulation time; on the contrary, when the attenuation factor is greater than the optimal attenuation factor, the imaging results of the MMV parametric model will get worse and worse with the increase of the coherent accumulation time. Obviously, this is because although the coherent accumulation time is further increased, the impact of the target motion on the imaging performance is not equal to the gain brought by the coherent processing, which makes the imaging result worse as a whole. Under different SNR conditions, the attenuation factors corresponding to the optimal imaging results are different. However, in a certain range, the imaging results are good. Therefore, selecting any value in this peripheral area can obtain ideal imaging results.

According to the analysis above, we take the attenuation factor as 0.1 , obtain the measurement number as 26 through calculation, and establish the MMV parametric model. To verify the superiority of the proposed method, we design a simulation experiment and compare it with the classical RCI based on SMV that is called the "traditional model." We use the mean square error (MSE) as a metric for quantifying reconstruction performance and carry out 100 Monte Carlo trials under different SNR conditions. The curves of MSE with varying SNR are given as shown in Figure 7. The imaging performance of the proposed method using the MMV parametric model is better than that of the classical method using the traditional model, and it is improved by around $7 \mathrm{~dB}$.

In order to intuitively reflect the imaging results and the advantages of the MSBL algorithm, we present the imaging results using different models and different reconstruction algorithms under the condition of $\mathrm{SNR}=10 \mathrm{~dB}$, as shown in Figure 7.

Figures $8(a)$ and $8(\mathrm{c})$ show the imaging results obtained by the traditional model, while Figures $8(\mathrm{~b})$ and $8(\mathrm{~d})$ show the imaging results obtained by the proposed model. By observing and comparing the MSEs of the imaging result, the side-lobe interferences of Figures 8(b) and 8(d) are lower than those of Figures 8(a) and 8(c), and the MSEs of Figures 8(b) and 8(d) are much smaller than those of Figures8(a) and8(c).Hence, the imaging performance of the proposed model is better than that of the traditional model. Furthermore, with the proposed model exploited, the MMV-OMP algorithm in [24] is compared with the MSBL algorithm, as shown in Figures 8(b) and 8(d). Because the MSE of Figure 8(d) is smaller than that of Figure 8(b), the imaging result obtained by MSBL is better than that obtained by MMV-OMP. It applies that MSBL can solve the proposed model better and obtain the better image based on the sparse feature of the model and the prior information of noise.

Therefore, according to two comparative experiments above, we can draw the conclusion that the proposed $\mathrm{Bi}$ RCI coherent processing method can improve imaging performance.

\section{Conclusions}

The Bi-RCI coherent processing method is focused on in this paper. Based on the parametric model extensively applied in $\mathrm{RCI}$, the MMV parametric model is proposed to realize BiRCI coherent processing. Considering the influence of the target translational motion and rotation in the imaging process, we give prominence to establish a model. Using the coherent part of mixed mode signals, the target distance variation rate is obtained by analyzing Doppler and microDoppler effects. Further, the coarse measurement number of the MMV parametric model is determined. Then, the MMV parametric model is established to realize coherent accumulation for the incoherent part of echoes. Besides, based on the existing algorithm, the MSBL algorithm is introduced to solve the MMV parametric model, and the image has been reconstructed finally. By simulations and 
comparisons, the validity and the superiority of the Bi-RCI coherent processing method are demonstrated.

In this paper, a feasible Bi-RCI coherent processing method is introduced, and it can improve the imaging performance of Bi-RCI. However, how to find the optimal solution of measurement number will be further studied in our future work. Here, the attenuation factor is introduced to select a coarse measurement number. Under this condition, we test the performance of the proposed method.

\section{Conflicts of Interest}

The authors declare that there is no conflict of interest regarding the publication of this paper.

\section{Acknowledgments}

This work was supported by the National Natural Science Foundation of China under Grants 61631019, 61971434, and 61801516 and the Natural Science Basic Research Program of Shaanxi Province under Grants 2018JM6072 and 2019JQ-238.

\section{References}

[1] H. Griffiths, "Developments in bistatic and networked radar," in Procedings of 2011 IEEE CIE International Conference on Radar, pp. 10-13, Chengdu, China, 2011.

[2] Y. Wang, S. Zhu, and J. Xu, "A range-ambiguous clutter suppression method for MIMO bistatic airborne radar," Journal of Radars, vol. 7, no. 2, pp. 202-221, 2018.

[3] X. Pan, D. Feng, Q. Fu, W. Wang, Y. Liu, and G. Wang, "On deception jamming for countering bistatic ISAR based on sub-Nyquist sampling," IET Radar, Sonar \& Navigation, vol. 8, no. 3, pp. 173-179, 2014.

[4] J. Chen, T. Zhang, J. Li, and X. Chen, "Joint sensor failure detection and corrupted covariance matrix recovery in bistatic MIMO radar with impaired arrays," IEEE Sensors Journal, vol. 19, no. 14, pp. 5834-5842, 2019.

[5] V. T. Vu and M. I. Pettersson, "Derivation of bistatic SAR resolution equations based on backprojection," IEEE Geoscience and Remote Sensing Letters, vol. 15, no. 5, pp. 694-698, 2018.

[6] Y. C. Lin, J. Y. Zhang, Y. Wu, and Q. Zhou, "Matrix inversion method for azimuth reconstruction in bistatic spaceborne high-resolution wide-swath SAR system," Journal of Radars, vol. 6, no. 4, pp. 388-396, 2017.

[7] S. Zhang, Y. Liu, and X. Li, "Bayesian bistatic ISAR imaging for targets with complex motion under low SNR condition," IEEE Transactions on Image Processing, vol. 27, no. 5, pp. 24472460, 2018.

[8] Y. Ma, E. W. Gill, and W. Huang, "Bistatic high-frequency radar ocean surface cross section incorporating a dualfrequency platform motion model," IEEE Journal of Oceanic Engineering, vol. 43, no. 1, pp. 205-210, 2018.

[9] M.-S. Kang, B.-S. Kang, S.-H. Lee, and K.-T. Kim, "BistaticISAR distortion correction and range and cross-range scaling," IEEE Sensors Journal, vol. 17, no. 16, pp. 5068-5078, 2017.

[10] Y. Jiang, S. Sun, T. S. Yeo, and Y. Yuan, "Bistatic ISAR distortion and defocusing analysis," IEEE Transactions on Aerospace and Electronic Systems, vol. 52, no. 3, pp. 1168-1182, 2016.
[11] B.-S. Kang, J.-H. Bae, M.-S. Kang, E. Yang, and K.-T. Kim, "Bistatic-ISAR cross-range scaling," IEEE Transactions on Aerospace and Electronic Systems, vol. 53, no. 4, pp. 19621973, 2017.

[12] L. Zhao, M. Gao, M. Martorella, and D. Stagliano, "Bistatic three-dimensional interferometric ISAR image reconstruction," IEEE Transactions on Aerospace and Electronic Systems, vol. 51, no. 2, pp. 951-961, 2015.

[13] D. Staglianò, S. Lischi, M. Martorella, and E. Giusti, "Bistatic three-dimensional interferometric ISAR," IET Radar, Sonar \& Navigation, vol. 10, no. 1, pp. 63-75, 2016.

[14] Y. Wang and X. Li, "Three-dimensional interferometric ISAR imaging for the ship target under the bi-static configuration," IEEE Journal of Selected Topics in Applied Earth Observations and Remote Sensing, vol. 9, no. 4, pp. 1505-1520, 2016.

[15] D. Li, X. Li, Y. Qin, Y. Cheng, and H. Wang, "Radar coincidence imaging: an instantaneous imaging technique with stochastic signals," IEEE Transactions on Geoscience and Remote Sensing, vol. 52, no. 4, pp. 2261-2277, 2014.

[16] X. Zhou, B. Fan, H. Wang, Y. Cheng, and Y. Qin, "Sparse Bayesian perspective for radar coincidence imaging with array position error," IEEE Sensors Journal, vol. 17, no. 16, pp. 52095219, 2017.

[17] S. Zhu, Y. He, H. Shi, A. Zhang, Z. Xu, and X. Dong, "Mixed mode radar coincidence imaging with hybrid excitation radar array," IEEE Transactions on Aerospace and Electronic Systems, vol. 54, no. 4, pp. 1589-1604, 2018.

[18] R. Li, Q. Zhang, L. H. Su, J. Liang, and Y. Luo, "Bistatic radar coincidence imaging based on sparse Bayesian learning," Journal of Electronics \& Information Technology, vol. 41, no. 12, pp. 2865-2872, 2019.

[19] X. R. Bai, G. C. Sun, Q. S. Wu, M. D. Xing, and Z. Bao, "Narrow-band radar imaging of spinning targets," Science China Information Sciences, vol. 54, no. 4, pp. 873-883, 2011.

[20] S. Zhu, A. Zhang, Z. Xu, and X. Dong, "Radar coincidence imaging with random microwave source," IEEE Antennas and Wireless Propagation Letters, vol. 14, pp. 1239-1242, 2015.

[21] Y. Luo, Y.-J. Chen, Y.-Z. Zhu, W.-Y. Li, and Q. Zhang, "Doppler effect and micro-Doppler effect of vortex-electromagneticwave-based radar," IET Radar, Sonar \& Navigation, vol. 14, no. 1, pp. 2-9, 2020.

[22] Z. Qun, H. Jian, L. Ying, and C. Yijun, "Research progresses in radar feature extraction, imaging, and recognition of target with micro-motions," Journal of Radars, vol. 7, no. 5, pp. 531-647, 2018.

[23] Z. Yang, L. Xie, and C. Zhang, "Off-grid direction of arrival estimation using sparse Bayesian inference," IEEE Transactions on Signal Processing, vol. 61, no. 1, pp. 38-43, 2013.

[24] Q.-Y. Liu, Q. Zhang, Y. Luo, K.-M. Li, and L. Sun, "Fast algorithm for sparse signal reconstruction based on off-grid model," IET Radar, Sonar \& Navigation, vol. 12, no. 4, pp. 390-397, 2018. 